\title{
Effect of Modified Sepiolite Nanofibers on Properties of cis-Polybutadiene Rubber Composite Nanomaterials
}

\author{
Fei Wang, ${ }^{1,2}$ Lei Feng, ${ }^{3}$ Qingguo Tang, ${ }^{1}$ Jinsheng Liang, ${ }^{1}$ Haifeng Liu, ${ }^{1}$ and Huimin Liu ${ }^{1}$ \\ ${ }^{1}$ Institute of Power Source \& Ecomaterials Science, Hebei University of Technology, 300130 Tianjin, China \\ ${ }^{2}$ Key Laboratory for Palygorskite Science and Applied Technology of Jiangsu Province, Huaiyin Institute of Technology, \\ Huaian 223003, China \\ ${ }^{3}$ Hebei United University Qianian College, 064400 Hebei, China
}

Correspondence should be addressed to Fei Wang; wangfei36777@126.com

Received 13 September 2013; Accepted 3 November 2013

Academic Editor: Baizeng Fang

Copyright (C) 2013 Fei Wang et al. This is an open access article distributed under the Creative Commons Attribution License, which permits unrestricted use, distribution, and reproduction in any medium, provided the original work is properly cited.

In order to use sepiolite nanofibers as cis-polybutadiene rubber fillers, the hydrophilic character of sepiolite nanofibers should be modified by grafting organic group and controlling surface free energy for improving compatibility of sepiolite nanofibers in rubber matrix. The relationship between the performance of the cis-polybutadiene rubber filled with sepiolite and the coupling modification was investigated, and the influence of coupling agent $\gamma$-(2,3-epoxypropoxy)propyltrimethoxysilane dosage on mechanical properties of cis-polybutadiene rubber materials was also studied. The results showed that the mechanical properties could be improved obviously after reinforcement by modified sepiolite nanofibers. The optimum dosage of coupling agent $\gamma$ (2,3-epoxypropoxy)propyltrimethoxysilane was 7\%, and the tensile strength and tearing strength increased by $108.3 \%$ and $74.1 \%$, respectively. On this basis, the reinforcement mechanism of the composite rubber materials was also discussed.

\section{Introduction}

Nowadays, cis-polybutadiene rubber materials play a critical role in many fields. Owing to the characteristics of cold tolerance, wear resistance, excellent elastic, age resistance, and so forth, cis-polybutadiene rubber materials have become a kind of useful product in the fields of rubber shoes, rubberized fabric, automotive sponge, and so forth [1-3]. However, cispolybutadiene rubber materials belong to organic materials, which have disadvantages of low compressive strength, high cost, and poor dimensional stability, especially not being able to form crystals at room temperature unless it is sufficiently stretched (stress-induced crystallization), and thus the stressinduced crystallization of cis-polybutadiene rubber materials is obviously lower than that of natural rubber [4-6]. Therefore, cis-polybutadiene rubber fillers have been developed in the recent years. The prime fillers in rubber industry such as carbon black production always make reinforced rubber deep color, which limits its applications in medical, sports, and domestic products. Moreover, the above fillers will consume large amounts of energy such as petroleum and natural gas and emit lots of heat and waste gas composed by the prime greenhouse gas of carbon dioxide and gaseous pollutants of sulfur dioxide and nitrogen oxide. This process would not only exacerbate tensions in the energy supply, but also pollute the environment [7-10].

Comparing with the former, the application of natural mineral materials as cis-polybutadiene rubber fillers will solve the serious environmental pollution problem. Sepiolite is a kind of porous mineral materials widely existing in nature in the form of fiber assemblies, leading to the limitation of usage [11-15]. Although the defibering treatment of sepiolite as a simple and cheap way to obtain mineral nanofibers is available for practical use, it has been less investigated except for a few exploratory researches which involve the destruction of its fibrous micromorphology by ball milling and the use of expensive and sensitive agents [16-20]. Therefore, the recent studies on sepiolite minerals reinforced composite materials have not fully explored the potential of sepiolite minerals [21-26], which provides promising future for further research. In our previous research, sepiolite 
nanofibers have been prepared using dry method airflow defibering technique [12]

In this paper, the cis-polybutadiene rubber composite materials were prepared using sepiolite mineral nanofibers as reinforced fillers, which have been reported scarcely in the literature. And their mechanical properties were also systematically investigated.

\section{Experimental Section}

The raw sepiolite samples which are one of natural fibrillar minerals were supplied by LB Nano-Material Technology Co., Ltd. The titanate coupling agent, NDZ-201 (isopropyl tri(dioctylpyrophosphate)titanate), and silane coupling agents, SG-Si998 (bis[-(triethoxysilyl)propyl]disulfide), KH560 ( $\gamma$-(2,3-epoxypropoxy)propyltrimethoxysilane), KH570 (3-(Methacryloyloxy)propyltrimethoxysilane), and KH590 (3-mercaptopropyltrimethoxysilane) were supplied by Shuguang Chemical Group Co., Ltd. The cis-polybutadiene rubber was supplied by Yanshan Petrochemical Co., Ltd. The compounding ingredients were purchased from chemical stores. $500.00 \mathrm{~g}$ of natural sepiolite samples were added into $3500 \mathrm{~mL}$ of water solution containing $175 \mathrm{~mL}$ of hydrochloric acid. Secondly, the mixture was stirred at speed of $2000 \mathrm{rpm}$ for $2 \mathrm{~h}$ by high-speed dispersing machine, and then the sepiolite solid was separated from the admixture by vacuum filtering and washed several times with distilled water until chlorine ion was not tested with silver nitrate, dried at $60^{\circ} \mathrm{C}$, and then the dry cake was crushed and ground into powders using the 20 mesh sieves. Then the above acid purified sepiolite samples were superfined by jet mill grinding. The grinding room air pressure is controlled between 0.7 and $0.85 \mathrm{MPa}$, the bearing protection air pressure is $0.2 \mathrm{MPa}$, the wash air pressure is $0.15 \mathrm{MPa}$, and the feeding rate is $6 \mathrm{~kg} / \mathrm{h}$ [12]. Contact angle of powder was measured using capillary penetration measurements. In this work, the powder was placed in a glass tube with a filter on the bottom. The tube was attached to an electrobalance (DataPhysics DCAT21), which could record the weight gain as a function of time when the bottom of the tube touched the testing solvent. The contact angle was calculated using the following equation:

$$
K \gamma_{\mathrm{lv}} \cos \theta=\left(\frac{\eta}{\rho^{2}}\right)\left(\frac{M^{2}}{t}\right)
$$

where $\gamma_{\mathrm{lv}}$ is the liquid-vapor interfacial tension, $\eta$ is the viscosity of the liquid, $\rho$ is the density of the liquid, $\theta$ is the contact angle which needs to be measured, and $M$ is the weight of the liquid which penetrates into the capillary. $K$ is a parameter contributed by capillary and is calculated by the $\theta$ of $\mathrm{n}$-Hexane whose contact angle can be considered as $0^{\circ}$. The surface energy (SE), dispersive interaction (Disp.), and polar interaction (Polar) were calculated using the contact angles of three kinds of liquid by Wu's equation:

$$
\gamma_{l}(1+\cos \theta)=\frac{4 \gamma_{s}^{d} \gamma_{l}^{d}}{\gamma_{s}^{d}+\gamma_{l}^{d}}+\frac{4 \gamma_{s}^{p} \gamma_{l}^{p}}{\gamma_{s}^{p}+\gamma_{l}^{p}}
$$

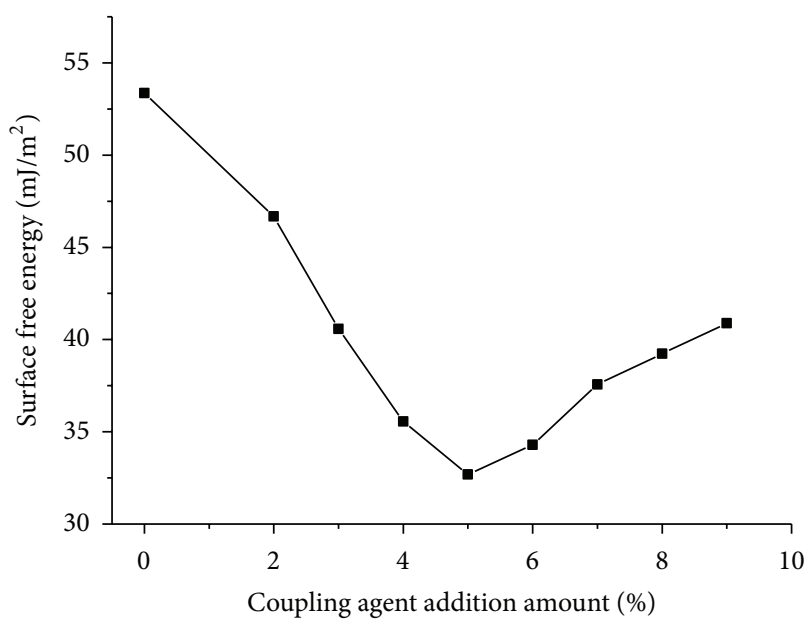

FIGURE 1: Variation of sepiolite nanofibers surface free energy after modification with different coupling agent ( $\gamma$-(2,3-epoxypropoxy) propyltrimethoxysilane) addition amounts.

where $\gamma_{l}$ is the liquid interfacial tension, $\gamma_{s}^{d}$ and $\gamma_{s}^{p}$ are the dispersive interaction and polar interaction of solid, respectively, $\gamma_{l}^{d}$ and $\gamma_{l}^{p}$ are the dispersive interaction and polar interaction of liquid, respectively, and $\theta$ is the contact angle which is measured before [27-29]. The rubber composites filled with sepiolite mineral nanofibers were prepared. cispolybutadiene rubber, modified sepiolite mineral nanofibers, and other compounding ingredients such as zinc oxide, stearic acid, zinc oxide, accelerator $\mathrm{CZ}$, antioxidant $\mathrm{RD}$, emollient, and sulphur were mixed using a laboratorysized two-roll mill. The optimum cure time $(t 90)$ which is the time for the completion of cure was determined at $145^{\circ} \mathrm{C}$ using a curometer. The composites were vulcanized at platen press with $13 \mathrm{MPa}$ pressure, based on the $t 90$ values. The microstructure of the samples was observed by scanning electron microscopy (Philips-XL30) at $25.0 \mathrm{kV}$ and $30 \mu \mathrm{A}$. Dumbbell shaped specimens were punched from the moulded sheets by a tensile specimen cutter. Tensile strength and elongation at break were measured following GB/T 5281998 using a universal tensile testing machine (CMT-6104).

\section{Results and Discussion}

Figure 1 shows the variation of surface free energy of sepiolite nanofibers after modification with different coupling agent addition $\quad(\gamma$-(2,3-epoxypropoxy)propyltrimethoxysilane) amounts.

From Figure 1, it can be seen that the sepiolite nanofibers after modification with the coupling agent ( $\gamma$-(2,3-epoxypropoxy)propyltrimethoxysilane) addition amount of $5 \%$ have the lowest surface free energy. When powders are dispersed into media, the lower the surface free energy, the better their dispersion [30-32]. According to the above analysis, $5 \%$ is selected as the coupling agent ( $\gamma$-(2,3-epoxypropoxy)propyltrimethoxysilane) addition amount for the sepiolite nanofibers in order to obtain the best modification effect of sepiolite nanofibers and the optimal properties of composite materials. 


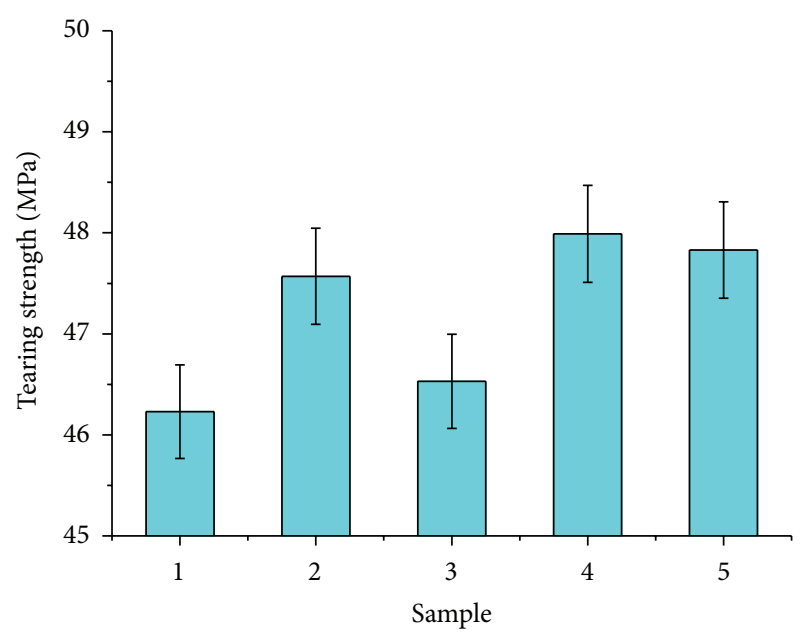

FIGURE 2: Effect of coupling agent for sepiolite nanofibers on tearing strength of rubber composite materials (1: KH-570, 2: KH-560, 3: KH-590, 4: NDZ-201, 5: Si-998; errors in tearing strength values $(y$ axis) are designated as the vertical error bars).

To study the influence of coupling agent for sepiolite nanofibers on mechanical properties of cis-polybutadiene rubber composite materials, five kinds of coupling agents for sepiolite nanofibers are chosen based on the above optimal coupling agent ( $\gamma$-(2,3-epoxypropoxy)propyltrimethoxysilane) addition amount. The tearing strength results of rubber composite materials are shown in Figure 2.

From Figure 2, we can see that the tearing strength values of rubber composite materials filled with sepiolite nanofibers modified with different kinds of coupling agent are close to each other. Among them, rubber composite materials filled with isopropyl tri(dioctylpyrophosphate)titanate modified sepiolite nanofibers have the best tearing strength. The reason for the phenomenon is mainly that isopropyl tri(dioctylpyrophosphate)titanate belongs to lipid coupling agent, which has relatively long chain and good toughness. Figure 3 shows the tensile strength results of cispolybutadiene rubber composite materials.

From Figure 3, it can be seen that the tensile strength of rubber composite materials filled by different kinds of coupling agent modified sepiolite nanofibers are different obviously. Among them, rubber composite materials filled with isopropyl tri(dioctylpyrophosphate)titanate modified sepiolite nanofibers have the lowest tensile strength. The reason for the phenomenon is mainly that isopropyl tri(dioctylpyrophosphate)titanate belongs to lipid coupling agent, which has physical winding rather than chemical bond $[33,34]$. The above results indicate that coupling agent modified sepiolite nanofibers could enhance the mechanical properties of rubber composite materials effectively. Moreover, rubber composite materials filled with sepiolite nanofibers modified by $\gamma$-(2,3-epoxypropoxy) propyltrimethoxysilane have the highest tensile strength. As shown in Figure 2, the rubber composite materials filled with $\gamma$-(2,3-epoxypropoxy)propyltrimethoxysilane and isopropyl tri(dioctylpyrophosphate)titanate modified sepiolite

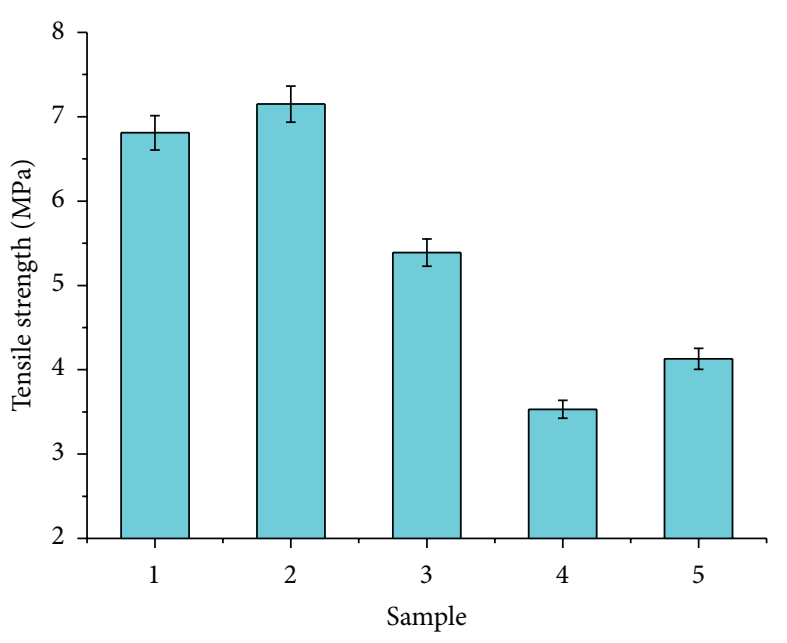

FIGURE 3: Effect of coupling agent for sepiolite nanofibers on tensile strength of rubber composite materials (1: KH-570, 2: KH-560, 3: KH-590, 4: NDZ-201, and 5: Si-998; errors in tensile strength values ( $y$-axis) are designated as the vertical error bars).

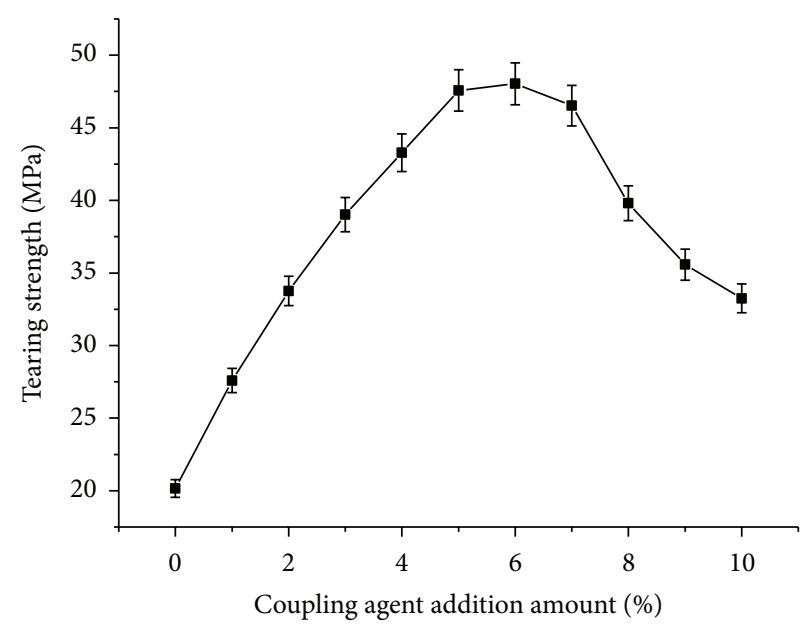

FIGURE 4: Variation of tearing strength of cis-polybutadiene rubber composite materials reinforced by sepiolite nanofibers modified by different coupling agent ( $\gamma$-(2,3-epoxypropoxy) propyltrimethoxysilane) addition amounts (errors in tearing strength values ( $y$-axis) are designated as the vertical error bars).

nanofibers have similar tearing strength. Therefore, $\gamma$-(2,3epoxypropoxy)propyltrimethoxysilane is chosen as the optimal coupling agent. Figure 4 shows the variation of tearing strength of cis-polybutadiene rubber composite materials reinforced by sepiolite nanofibers modified by different coupling agent $(\gamma$-(2,3-epoxypropoxy)propyltrimethoxysilane) addition amounts.

From Figure 4, we can see that the tearing strength values of rubber composite materials filled with different coupling agent addition amounts are different obviously. Among them, rubber composite materials filled with $6 \%$ $\gamma$-(2,3-epoxypropoxy)propyltrimethoxysilane modified sepiolite nanofibers have the best tearing strength. Figure 5 shows the tensile strength results of cis-polybutadiene rubber composite materials. 


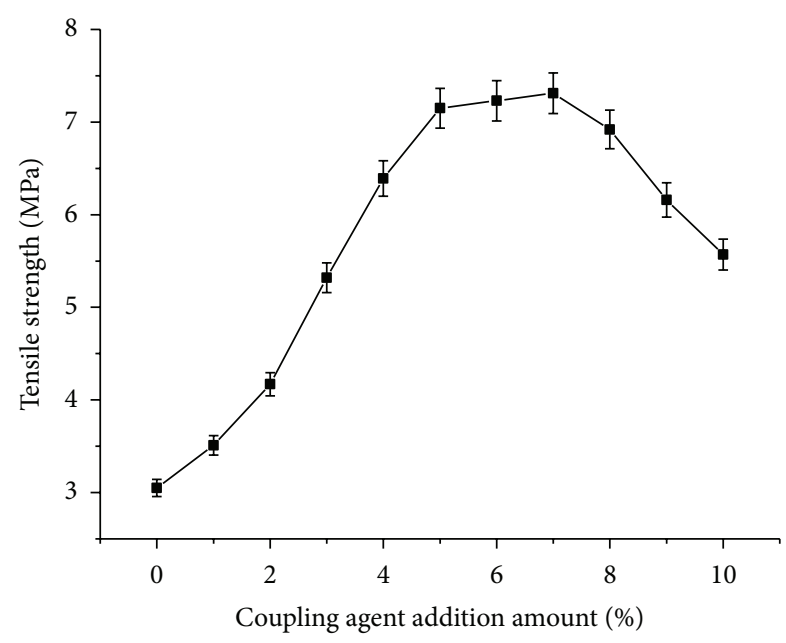

FIGURE 5: Variation of tensile strength of cis-polybutadiene rubber composite materials reinforced by sepiolite nanofibers modified by different coupling agent ( $\gamma$-(2,3-epoxypropoxy)propyltrimethoxysilane) addition amounts (errors in tensile strength values ( $y$-axis) are designated as the vertical error bars).

From Figure 5, we can see that the tensile strength values of rubber composite materials filled with different coupling agent addition amounts are different obviously. Among them, rubber composite materials filled with $7 \% \gamma$ (2,3-epoxypropoxy)propyltrimethoxysilane modified sepiolite nanofibers have the best tensile strength. As shown in Figure 4 , the rubber composite materials filled with $6 \%$ and $7 \% \gamma$-(2,3-epoxypropoxy)propyltrimethoxysilane modified sepiolite nanofibers have similar tearing strength. Therefore, $7 \% \gamma$-(2,3-epoxypropoxy)propyltrimethoxysilane is chosen as the optimal coupling agent amount. The main reason for this phenomenon lies in the good dispersion of modified sepiolite nanofibers in the cis-polybutadiene rubber matrix at a nanometer scale and the strong interaction between sepiolite nanofibers and cis-polybutadiene rubber, which exhibits nanometer effect and physical crosslink of sepiolite nanofibers. Due to transferring stress and limiting the cracks expansion of sepiolite nanofibers, the modified sepiolite nanofibers can improve the mechanical properties of cispolybutadiene rubber.

It is known that the dispersion of the fillers in the polymer matrix has significant effect on the mechanical properties of the composites $[35,36]$. A good dispersion can be achieved by surface modification of the filler particles and appropriate processing conditions. As shown in Figure 6, the dispersibility of modified sepiolite nanofibers in the cis-polybutadiene rubber matrix is improved obviously compared with the unmodified ones (Figure 6(a)), and the average diameter of modified sepiolite nanofibers is less than $100 \mathrm{~nm}$. For the cis-polybutadiene rubber nanocomposites containing $7 \% \quad \gamma$-(2,3-epoxypropoxy)propyltrimethoxysilane modified sepiolite nanofibers shown in Figure 6(b), most sepiolite nanofibers aggregates are broken down to primary particles, which should maximize the interfacial interaction between the sepiolite nanofibers and the polymer matrix.

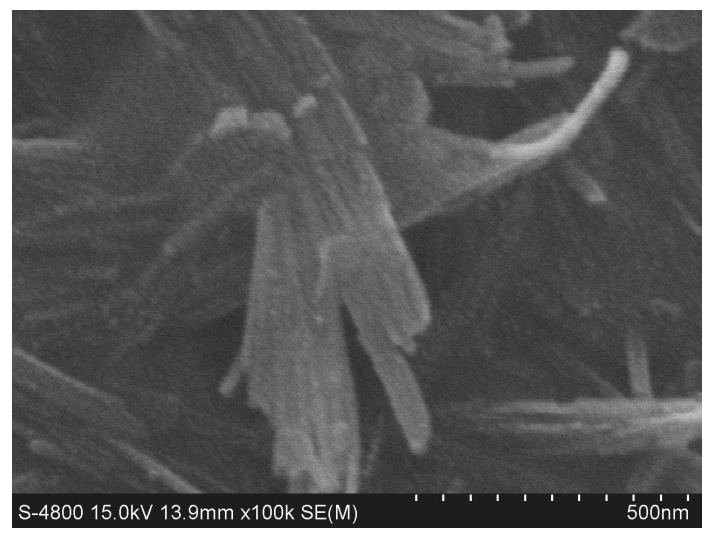

(a)

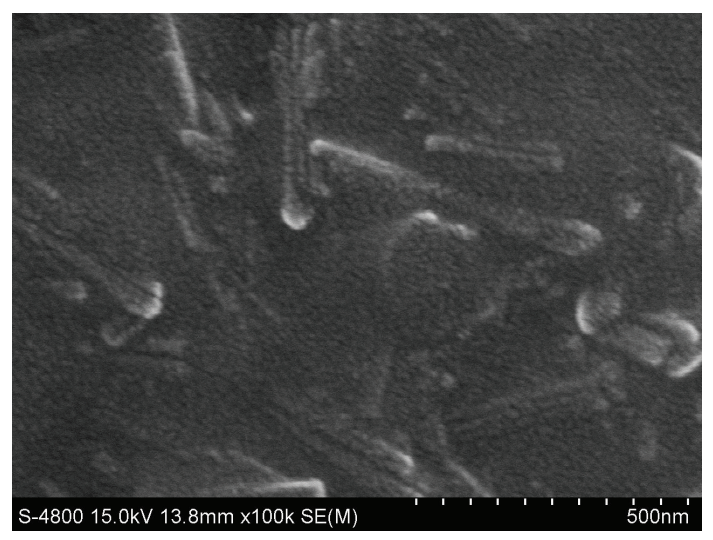

(b)

FIGURE 6: SEM micrograph of fracture surface of nanocomposites ((a) containing unmodified sepiolite samples; (b) containing sepiolite nanofibers modified by $7 \% \gamma$-(2,3-epoxypropoxy) propyltrimethoxysilane).

\section{Conclusions}

The hydrophilic character of sepiolite nanofibers should be modified by grafting organic group and controlling surface energy for improving compatibility of sepiolite nanofibers in rubber matrix, in order to use sepiolite nanofibers as cis-polybutadiene rubber fillers. It can be concluded that the mechanical properties can be improved obviously after reinforcement by modified sepiolite nanofibers. The tensile strength and tearing strength increased by $108.3 \%$ and $74.1 \%$, respectively, when the optimum dosage of coupling agent $\gamma$-(2,3-epoxypropoxy)propyltrimethoxysilane was $7 \%$. Microstructure of cis-polybutadiene rubber composite materials fracture surface exhibits nanometer effect and physical crosslink of sepiolite nanofibers, which should maximize the interfacial interaction between the sepiolite nanofibers and the polymer matrix.

\section{Acknowledgments}

This research was financially supported by the Application Foundation and Advanced Technology Research Program of Tianjin, China (Grant no. 12JCQNJC02100). 


\section{References}

[1] V. K. Srivastava, M. Maiti, and R. V. Jasra, "Synthesis and utilization of alternative chain transfer agent in cobalt catalyzed 1,3-butadiene polymerization reaction to produce cispolybutadiene rubber," European Polymer Journal, vol. 47, no. 12, pp. 2342-2350, 2011.

[2] G. J. Song, Z. Gu, P. Y. Li, L. Wang, and L. Gao, "The properties of organo-montmorillonite/ cis-1,4-polybutadiene rubber nanocomposites and the effect of recovered solvent on the conversion of butadiene polymerization," Applied Clay Science, vol. 65, pp. 158-161, 2012.

[3] Y. Hu, Z. Jia, Y. Li, L. Chang, and Y. Wang, "Synthesis and impact properties of in situ bulk made ABS resins toughened by high cis-1,4 polybutadiene," Materials Science and Engineering A, vol. 528, no. 22-23, pp. 6667-6672, 2011.

[4] A. Nimpaiboon and J. Sakdapipanich, "A model study on effect of glucose on the basic characteristics and physical properties of natural rubber," Polymer Testing, vol. 32, pp. 1408-1416, 2013.

[5] S. S. Sarkawi, W. K. Dierkes, and J. W. M. Noordermeer, "The influence of non-rubber constituents on performance of silica reinforced natural rubber compounds," European Polymer Journal, vol. 49, pp. 3199-3209, 2013.

[6] J. Bartoš, H. Švajdlenková, Y. Yu, G. Dlubek, and R. KrauseRehberg, "Molecular probe dynamics and free volume in glass-formers: 1,2- and 1,4-poly(butadiene)s," Chemical Physics Letters, vol. 584, pp. 88-92, 2013.

[7] X. Li, Y. Xia, Z. Li, and Y. Xia, "Three-dimensional numerical simulations on the hyperelastic behavior of carbon-black particle filled rubbers under moderate finite deformation," Computational Materials Science, vol. 55, pp. 157-165, 2012.

[8] M. A. Hassan, A. Abouel-Kasem, M. A. El-Sharief, and F. Yusof, "Evaluation of the material constants of nitrile butadiene rubbers (NBRs) with different carbon black loading (CB): FEsimulation and experimental," Polymer, vol. 53, pp. 3807-3814, 2012.

[9] J. L. Diani, P. Gilormini, Y. Merckel, and F. Vion-Loisel, "Micromechanical modeling of the linear viscoelasticity of carbon-black filled styrene butadiene rubbers: the role of the filler-rubber interphase," Mechanics of Materials, vol. 59, pp. 6572, 2013.

[10] S. Vinayasree, M. A. Soloman, V. Sunny, P. Mohanan, P. Kurian, and M. R. Anantharaman, "A microwave absorber based on strontium ferrite-carbon black-nitrile rubber for $\mathrm{S}$ and X-band applications," Composites Science and Technology, vol. 82, pp. 69-75, 2013.

[11] M. Suarez and E. Garcia-Romero, "Variability of the surface properties of sepiolite," Applied Clay Science, vol. 67-68, pp. 7282, 2012.

[12] F. Wang, J. Liang, Q. Tang, L. Li, and L. Han, "Preparation and far infrared emission properties of natural sepiolite nanofibers," Journal of Nanoscience and Nanotechnology, vol. 10, no. 3, pp. 2017-2022, 2010.

[13] E. Işçi and S. I. Turutoğlu, "Stabilization of the mixture of bentonite and sepiolite as a water based drilling fluid," Journal of Petroleum Science and Engineering, vol. 76, no. 1-2, pp. 1-5, 2011.

[14] B. F. Jones and K. M. Conko, "Environmental influences on the occurrences of sepiolite and palygorskite: a brief review," Developments in Clay Science, vol. 3, pp. 69-83, 2011.

[15] J. Hrenovic, Z. Z. Gobac, and V. Bermanec, "Occurrence of sepiolite in Croatia and its application in phosphate removal from wastewater," Applied Clay Science, vol. 59-60, pp. 64-68, 2012.

[16] H. Yin, J.-S. Liang, Q.-G. Tang, G.-C. Liang, L.-J. Wang, and G.-S. Li, "Influence of sepiolite microstructure on thermal insulation properties of coating material," Journal of Synthetic Crystals, vol. 34, no. 3, pp. 519-524, 2005.

[17] N. Volle, F. Giulieri, A. Burr, S. Pagnotta, and A. M. Chaze, "Controlled interactions between silanol groups at the surface of sepiolite and an acrylate matrix: consequences on the thermal and mechanical properties," Materials Chemistry and Physics, vol. 134, no. 1, pp. 417-424, 2012.

[18] S. Tunç, O. Duman, and A. Çetinkaya, "Electrokinetic and rheological properties of sepiolite suspensions in the presence of hexadecyltrimethylammonium bromide," Colloids and Surfaces A, vol. 377, no. 1-3, pp. 123-129, 2011.

[19] N. Volle, F. Giulieri, A. Burr, S. Pagnotta, and A. M. Chaze, "Controlled interactions between silanol groups at the surface of sepiolite and an acrylate matrix: consequences on the thermal and mechanical properties," Materials Chemistry and Physics, vol. 134, no. 1, pp. 417-424, 2012.

[20] Q. Zhu, Y. Zhang, F. Lv, P. K. Chu, Z. Ye, and F. Zhou, "Cuprous oxide created on sepiolite: preparation, characterization, and photocatalytic activity in treatment of red water from 2,4,6trinitrotoluene manufacturing," Journal of Hazardous Materials, vol. 217-218, pp. 11-18, 2012.

[21] A. Mejía, N. García, J. Guzmán, and P. Tiemblo, "Confinement and nucleation effects in poly(ethylene oxide) meltcompounded with neat and coated sepiolite nanofibers: modulation of the structure and semicrystalline morphology," European Polymer Journal, vol. 49, pp. 118-129, 2013.

[22] D. J. Huang, B. Mu, and A. Q. Wang, "Preparation and properties of chitosan/poly (vinyl alcohol) nanocomposite films reinforced with rod-like sepiolite," Materials Letters, vol. 86, pp. 69-72, 2012.

[23] P. Verge, T. Fouquet, C. Barrère, V. Toniazzo, D. Ruch, and A. S. Bomfim, "Organomodification of sepiolite clay using bio-sourced surfactants: compatibilization and dispersion into epoxy thermosets for properties enhancement," Composites Science and Technology, vol. 79, pp. 126-132, 2013.

[24] M. Lombardi, P. Fino, G. Malucelli, and L. Montanaro, "Exploring composites based on PPO blend as ablative thermal protection systems-part I: the role of layered fillers," Composite Structures, vol. 94, no. 3, pp. 1067-1074, 2012.

[25] H. Chen, H. Lu, Y. Zhou, M. Zheng, C. Ke, and D. Zeng, "Study on thermal properties of polyurethane nanocomposites based on organo-sepiolite," Polymer Degradation and Stability, vol. 97, no. 3, pp. 242-247, 2012.

[26] G. Ahmetli, H. Deveci, U. Soydal, A. Seker, and R. Kurbanli, "Coating, mechanical and thermal properties of epoxy toluene oligomer modified epoxy resin/sepiolite composites," Progress in Organic Coatings, vol. 75, pp. 97-105, 2012.

[27] X. Dong, Q. Y. Zong, and J. X. He, "Anisotropic surface properties and wettability of disperse dye single crystal," Dyes and Pigments, vol. 96, pp. 636-641, 2013.

[28] B. P. Li, Y. H. Zhang, and G. Z. Wu, "Thermoplastics reinforced by self-welded glass fibers: effect of interfacial affinity on preferential segregation," Polymer, vol. 54, pp. 2440-2449, 2013.

[29] C. Weber and H. Stanjek, "Energetic and entropic contributions to the work of adhesion in two-component, three-phase solidliquid-vapour systems," Colloids and Surfaces A, vol. 441, pp. 331-339, 2014. 
[30] B. P. Singh, S. Nayak, S. Samal, S. Bhattacharjee, and L. Besra, "The role of poly(methacrylic acid) conformation on dispersion behavior of nano $\mathrm{TiO}_{2}$ powder," Applied Surface Science, vol. 258, no. 8, pp. 3524-3531, 2012.

[31] A. Najafi, F. Golestani-Fard, H. R. Rezaie, and N. Ehsani, "Effect of APC addition on precursors properties during synthesis of $\mathrm{B}_{4} \mathrm{C}$ nano powder by a sol-gel process," Journal of Alloys and Compounds, vol. 509, no. 37, pp. 9164-9170, 2011.

[32] B. Benli, H. Du, and M. S. Celik, "The anisotropic characteristics of natural fibrous sepiolite as revealed by contact angle, surface free energy, AFM and molecular dynamics simulation," Colloids and Surfaces A, vol. 408, pp. 22-31, 2012.

[33] S. Kango, S. Kalia, A. Celli, J. Njuguna, Y. Habibi, and R. Kumar, "Surface modification of inorganic nanoparticles for development of organic-inorganic nanocomposites-a review," Progress in Polymer Science, vol. 38, pp. 1232-1261, 2013.

[34] N. Wang, Q. Fang, J. Zhang, E. Chen, and X. Zhang, "Incorporation of nano-sized mesoporous MCM-41 material used as fillers in natural rubber composite," Materials Science and Engineering A, vol. 528, no. 9, pp. 3321-3325, 2011.

[35] J. H. Kim, J. H. Lee, J. Y. Min et al., "Cryomilling effect on the mechanical alloying behaviour of ferritic oxide dispersion strengthened powder with $\mathrm{Y}_{2} \mathrm{O}_{3}$," Journal of Alloys and Compounds, vol. 580, pp. 125-130, 2013.

[36] N. Jouault, F. Dalmas, F. Boué, and J. Jestin, "Multiscale characterization of filler dispersion and origins of mechanical reinforcement in model nanocomposites," Polymer, vol. 53, no. 3, pp. 761-775, 2012. 

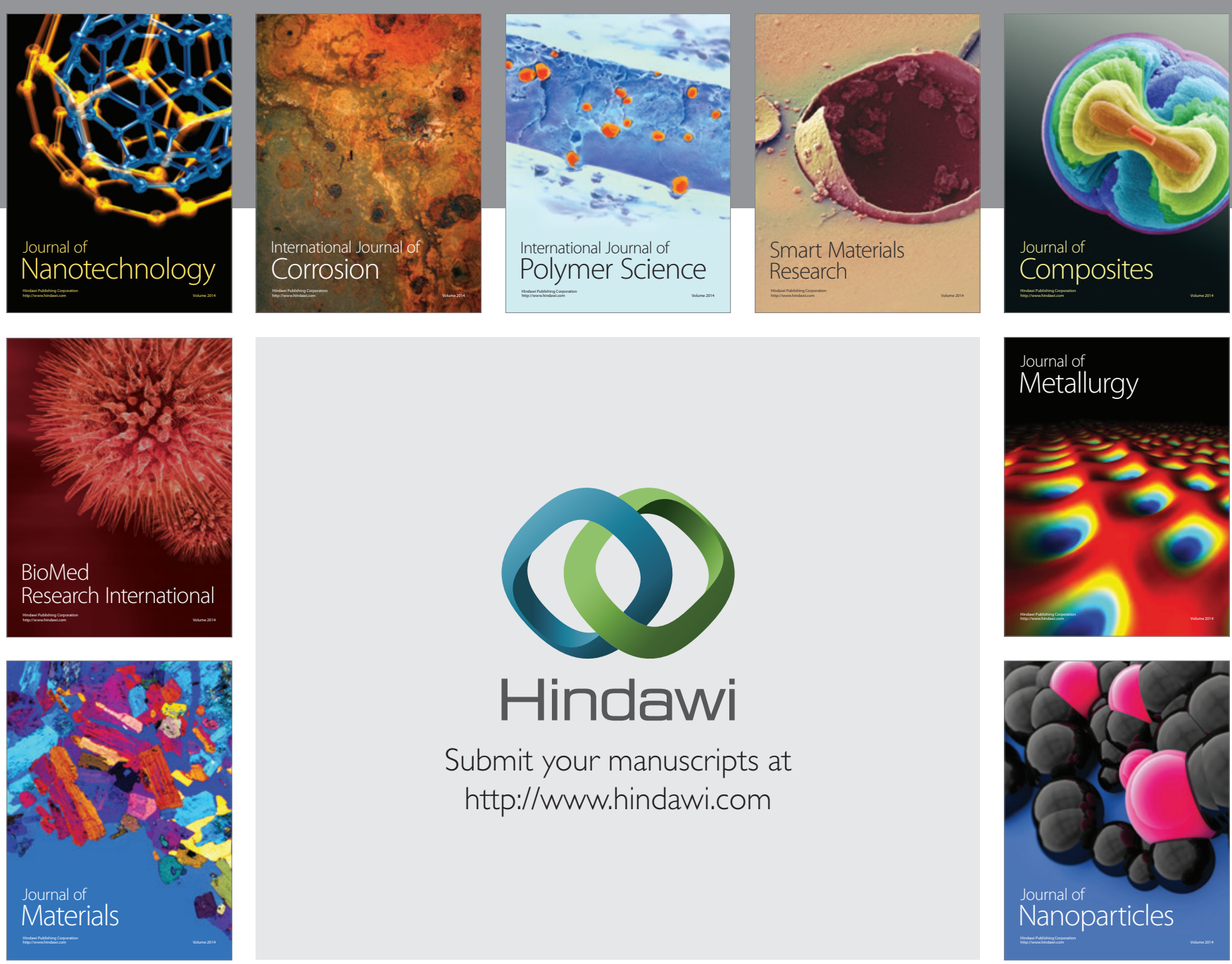

Submit your manuscripts at http://www.hindawi.com
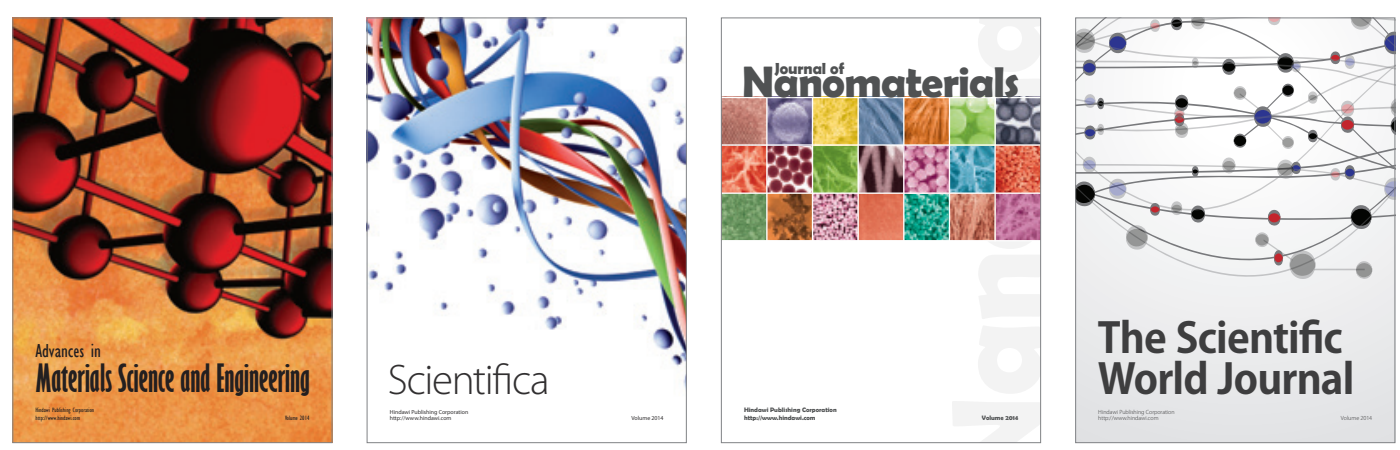

\section{The Scientific World Journal}
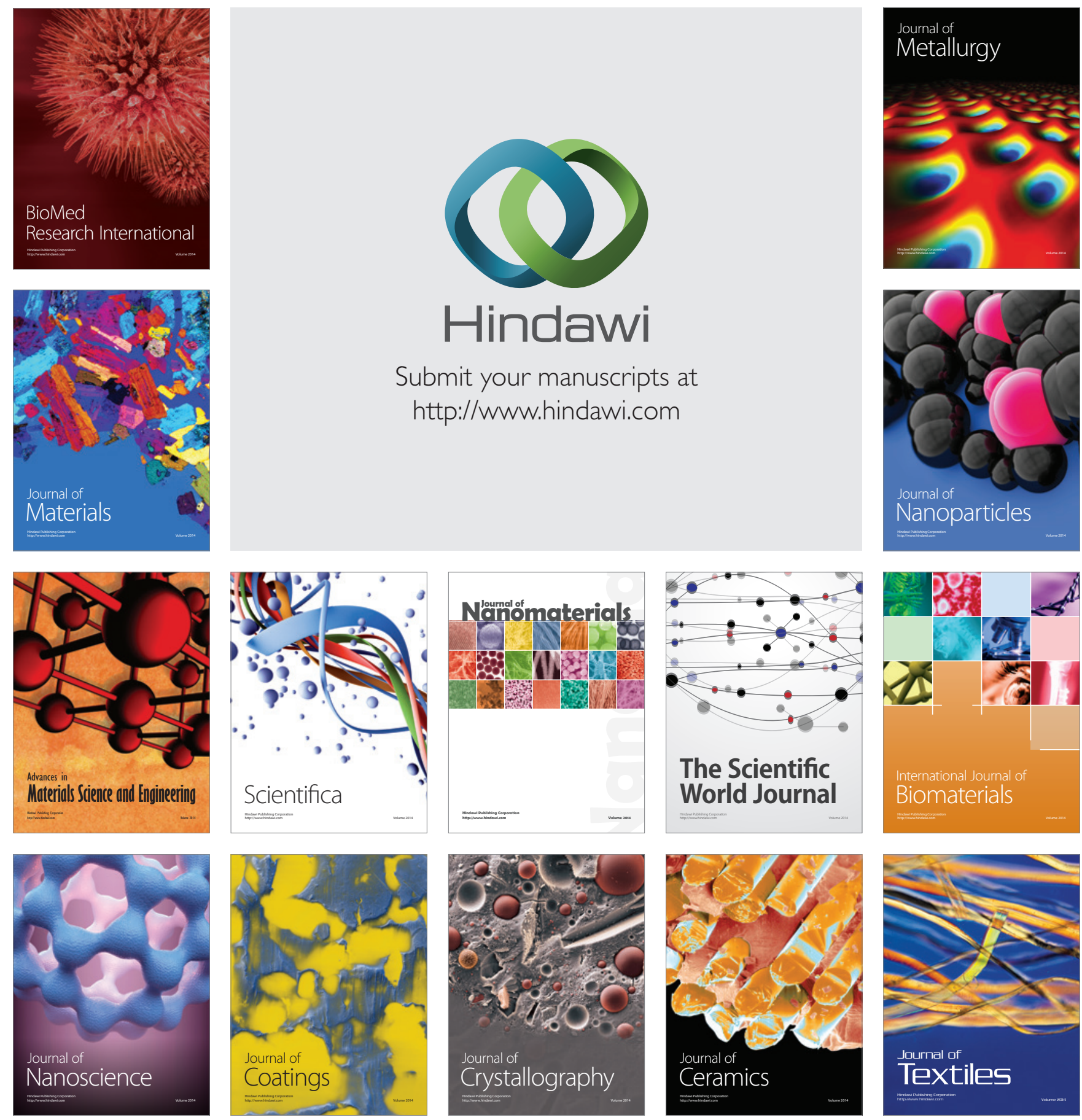